\title{
Post-Neoadjuvant Therapy
}

\author{
Michael Untch ${ }^{a}$ Andreas Schneeweiss ${ }^{b}$ David Krug ${ }^{c}$ Thorsten Kühn ${ }^{d}$ \\ Sibylle Loible \\ ${ }^{a}$ Frauenklinik / Interdisziplinäres Brustzentrum, HELIOS Klinikum Berlin-Buch, Berlin, Germany; ${ }^{b}$ Nationales Zentrum \\ für Tumorerkrankungen, Universitätsklinikum Heidelberg, Heidelberg, Germany; ${ }^{{ }^{C} K l i n i k}$ für Strahlentherapie \\ (Radioonkologie), Universitätsklinikum Schleswig-Holstein, Kiel, Germany; ${ }^{\mathrm{d}}$ Frauenklinik, Städtische Kliniken \\ Esslingen a.N., Esslingen, Germany; ${ }^{e}$ GBG Forschungs GmbH, Neu-Isenburg, Germany
}

\section{Question 1: One of the Most Exciting Presentations at the San Antonio Breast Cancer Symposium in San Antonio in 2018 Was the KATHERINE Study. What Is the Key Message from the KATHERINE Study Concerning Neoadjuvant and Post-Neoadjuvant Treatment for Early HER2-neu-Overexpressing Breast Cancer?}

Schneeweiss: Improvement of patient outcome by stratifying the adjuvant treatment according to the pathological response to the neoadjuvant systemic treatment is possible not only in patients with HER2-negative early breast cancer (EBC) (CREATE-X, POETIC) but also in patients with HER2-positive EBC. Therefore, the main reason to give systemic treatment in the neoadjuvant setting is no longer to reduce morbidity by de-escalating locoregional treatment but to utilize in vivo sensitivity testing to increase the probability of cure.

Krug: Although neoadjuvant treatment does not improve prognosis per se, it offers the possibility to stratify patients for escalation or de-escalation of further treatment and evaluation of new drugs or treatment strategies.
This study adds significant weight to the validity of the neoadjuvant treatment approach. It shows that subtypeand response-tailored trials in the post-neoadjuvant setting are feasible and may deliver practice-changing results.

Kühn: The KATHERINE study provides an important proof of principle, i.e., that response to chemotherapy is a promising tool which allows tailoring of treatment decisions and may serve as a dynamic biomarker to select patients who require an escalation of systemic treatment. We urgently await data from the PENELOPE study whether this concept can also be translated to luminal tumors. On the other hand, strategies of de-escalation of locoregional treatment in patients with a good response to primary systemic treatment are awaited from the B 51 study for locoregional radiotherapy after neoadjuvant therapy and surgery.

Loibl: T-DM1 improves disease-free survival (DFS) in HER2-positive breast cancer patients with residual disease after neoadjuvant therapy.

\section{KARGER}

() 2019 S. Karger AG, Basel
Prof. Dr. med. Michael Untch

E-Mail michael.untch@helios-kliniken.de 
Question 2: What Is the Optimal Post-Neoadjuvant Treatment in Patients Who Achieved a Pathologic Complete Remission after Neoadjuvant

Chemotherapy and a Double Antibody Combination with Trastuzumab and Pertuzumab? Should

These Patients Have a De-Escalation from a

Double Antibody Combination to Single Antibody

(Trastuzumab) or Should They Be Treated with a

Post-Neoadjuvant Double Antibody Combination?

And for How Many Cycles?

Schneeweiss: This is an open question. Currently, no data from randomized clinical trials are available addressing this question. Given the results of APHINITY that prove the superior efficacy regarding 3-year invasive DFS of dual antibody treatment for one year in high-risk EBC patients in the adjuvant setting, I would recommend to also complete dual antibody treatment in case of pathologic complete remission (pCR) following neoadjuvant chemotherapy plus dual antibodies for up to one year. Nevertheless, it would be very interesting to know whether de-escalating the adjuvant treatment to only trastuzumab (or even no adjuvant anti-HER2 treatment) in case of pCR is equally effective.

Krug: This is an interesting question since the value of additional systemic treatment after achieving pCR is not really clear. Does it matter how we achieve pCR? This is also relevant in the context of several trials showing excellent pCR rates in triple-negative breast cancer (TNBC) patients using platinum-taxane combinations $[1,2]$. Do all of these patients need adjuvant anthracycline-based chemotherapy as is usually given in these trials? I would recommend to use dual antibody treatment in case of pCR in node-positive patients and in those with negative hormone receptor status, since these were the subgroups which benefited most in the APHINITY trial. Apart from that, a clinical trial studying de-escalation in this setting would be really interesting.

Kühn: According to the result of the APHINITY trial, we currently continue the dual blockade in estrogen receptor-negative patients and in women who presented initially with a positive lymph node status. This strategy requires confirmation, since it is unclear whether the small benefit that was observed in these subgroups translates into a clinical benefit in patients who achieve pCR.

Loibl: In patients without nodal involvement at the time of primary diagnosis, trastuzumab alone seems sufficient. Patients with nodal involvement should receive the dual blockade.
Question 3: For Patients with TNBC, Neoadjuvant

Treatment Is Choice Number 1. (a) Is Platin Standard in the Neoadjuvant Treatment of Patients with TNBC? (b) If for any Reasons Platin Has Not Been Given as Part of the Neoadjuvant Treatment, What Is Your First Choice for the Systemic Post-Neoadjuvant Treatment? Platinum or Capecitabine?

Schneeweiss: (a) Platinum compounds can improve pCR rates by about $15-20 \%$. However, no predictive marker is currently available for a response to a platinum compound in the neoadjuvant setting, and the addition of carboplatin to a complete anthracycline/taxane/cyclophosphamide-containing neoadjuvant regimen has not improved event-free survival (CALCGB trial). Therefore, in my opinion, platinum compounds are not indicated in all patients with early TNBC but only in high-risk patients, e.g., patients with axillary lymph node involvement or with insufficient response to the first anthracycline/cyclophosphamide-containing part of the neoadjuvant treatment.

(b) My first choice is capecitabine because currently only for capecitabine are there data of a randomized phase III trial (CREATE-X) available. In addition, as already mentioned, no predictive marker for better efficacy of one or the other agent is available.

Krug: (a) The addition of carboplatin to anthracycline/ taxane-based neoadjuvant chemotherapy improves $\mathrm{pCR}$ rates, but it certainly also adds significant toxicity, as shown in several randomized controlled trials $[3,4]$. In the GeparSixto trial, DFS was significantly improved by the addition of carboplatin [5]; however, this was not shown in the final CALGB 40603 data presented at this year's ASCO meeting [6]. I think that the addition of carboplatin is certainly justified in higher risk patients; however, I would not see it as mandatory in early-stage nodenegative TNBC.

(b) As there is no level 1 evidence for the use of platinum in the post-neoadjuvant setting, I would prefer capecitabine.

Kühn: (a) Most patients with small tumors $(<1 \mathrm{~cm})$ undergo primary surgery at our institution. The addition of carboplatin to anthracycline/taxane-based chemotherapy is the standard for most patients with triple-negative tumors. The omission of carboplatin is considered for patients with an unfavorable performance status, those with a high toxicity under chemotherapy, or patients with an excellent response after 4 cycles of EC.

(b) Patients who do not achieve a complete response receive capecitabine according to the CREATE $\mathrm{X}$ trial.

Loibl: (a) Yes. (b) Capecitabine. 


\section{Question 4: If You Give Additional Capecitabine to Your TNBC Patients with Residual Disease after Optimal Neoadjuvant Chemotherapy, Do You Recommend Concomitant or Sequential Locoregional Radiotherapy? And If You Recommend Concomitant Therapy, Do You De-Escalate the Dose of Capecitabine?}

Schneeweiss: I prefer the sequence of capecitabine followed by locoregional radiotherapy because I believe that the risk of developing distant metastases due to persistent micrometastatic disease determines the ultimate prognosis. Furthermore, systemic treatment is most effective if it can be administered in full dose. Radiotherapy given in parallel potentially leads to more frequent dose reductions which in turn can impact efficacy. In addition, systemic treatment can also control locoregional micometastases. Therefore, I prefer to complete systemic treatment before starting locoregional radiotherapy.

Krug: In the CREATE-X-trial [7], capecitabine was given sequentially at a dose of $1,250 \mathrm{mg} / \mathrm{m}^{2}$ b.i.d. days 1-14 every 3 weeks either before or after administration of locoregional radiotherapy. Approximately $70 \%$ of the patients in both arms of the trial received radiotherapy. Patients with TNBC and residual disease after neoadjuvant chemotherapy often face a high risk of locoregional and distant recurrence. Delaying radiotherapy due to the delivery of post-neoadjuvant chemotherapy might increase the risk of locoregional recurrence and vice versa if radiotherapy is delivered first. Administering concomitant chemoradiotherapy seems like the logical choice in this situation; however, there are several caveats. As capecitabine is a potent radiosensitizer, even giving it at a lower dose of $825 \mathrm{mg} / \mathrm{m}^{2}$ b.i.d. considerably increased radiotherapy-related side effects in a prospective phase II trial and led to a dose modification [8]. Using hypofractionated radiotherapy decreases the time of concomitant treatment; however, this is still not considered standard in case of regional nodal irradiation and post-mastectomy radiotherapy. A reduction of the capecitabine dose and dose density might reduce the treatment benefit seen in the CREATE-X-trial. Thus, I would consider concomitant chemoradiotherapy only in patients with a very high risk of locoregional recurrence, especially in the case of positive margins or residual irresectable disease.

Kühn: The CREATE X trial showed a survival benefit in this group of patients. Therefore, at our institute, capecitabine is offered to all patients who do not achieve pCR. The individual benefit is, however, discussed with the patients and weighed up against the risks. This relates especially to patients who are reluctant to continue chemotherapy because of a high toxicity experienced during preceding treatment. The CREATE X trial is not clear regarding these issues because the patients were treated in both settings, and no data comparing both treatment strategies were published. In our clinical practice, this decision is largely dependent on the performance status of the patient and the hematotoxicity of the preceding chemotherapy. In most patients, we perform sequential radiotherapy. Capecitabine can then be started at a standard dose.

Loibl: No, but capecitabine is given to selected patients only.

\section{Question 5: What Is the Treatment of Choice in Patients Who Had Had Histologically Proven Ipsilateral Axillary Lymph Node Involvement before Neoadjuvant Therapy and Achieved a Clinical and Radiological Complete Remission: Full Axillary Lymph Node Dissection, Sentinel Node Biopsy with Frozen Section, or Targeted Axillary Dissection?}

Schneeweiss: Given the currently available data, this depends on the willingness of the patient to accept some uncertainties for less locoregional morbidity. If the patient is willing to do so following thorough counseling, I prefer to do targeted axillary dissection. Otherwise I would recommend complete axillary dissection following neoadjuvant chemotherapy.

Krug: Several prospective randomized controlled trials have addressed the use of sentinel lymph node biopsy after neoadjuvant therapy in patients with clinically involved lymph nodes before initiation of treatment. False negative rates (FNR) are generally above $10 \%$ for unselected patients. Data on recurrence rates are only available from cohort studies. The results of targeted axillary dissection in the MD Anderson cohort are impressive [9]; however, there are still many unanswered questions regarding reproducibility in a broader setting. I would still regard axillary lymph node dissection as the standard of care in the above-mentioned setting; however, the tide is changing, and it remains crucial to conduct clinical trials.

Kühn: Four procedures are performed in clinical practice: full axillary lymph node dissection, sentinel lymph node biopsy, targeted lymph node biopsy, and targeted axillary dissection. So far, no data are available that compare these procedures with regard to outcome (DFS), morbidity, and costs. Axillary dissection appears to be an overtreatment especially in view of the increasing number of patients with pCR in the lymph nodes. Targeted axillary dissection (removal of a clipped node combined with a sentinel lymph node biopsy) is associated with the 
most favorable FNR. It is, however, unclear whether the higher FNR observed for the sentinel lymph node biopsy procedure translates into higher recurrence rates and whether the high technical effort associated with localization techniques (especially with the use of clips and needles) is associated with a clinical benefit. The optimal localization technique for the target lymph node is currently under discussion. A Europe-wide register is planned to gather data for the different procedures and hopefully define the best.

Loibl: Either sentinel node biopsy or targeted axillary dissection, depending on the type of confirmation of the diagnosis for the involved lymph nodes in the axilla.

\section{Question 6: What Is the Post-Neoadjuvant Locoregional Radiotherapy Treatment of Choice for Patients Who Had One or Two Histologically Involved Axillary Nodes before Neoadjuvant Treatment and Had Clinical, Radiological, and Histopathological Complete Remission after Neoadjuvant Treatment?}

Schneeweiss: Currently, at our center, we follow the guidelines for locoregional radiotherapy in patients with 1-2 involved axillary nodes who had no neoadjuvant chemotherapy but initial surgery. However, I really hope that the currently recruiting NSABP B-51 trial will help to answer this important question in the near future, in a way that we can de-escalate locoregional radiotherapy in those patients without compromising outcome.

Krug: The optimal locoregional strategy in patients receiving neoadjuvant treatment is a matter of intense debate. Until now, no data from prospective randomized controlled trials studying the tailoring of adjuvant radiotherapy based on treatment response have been published, and it will take several more years until results from trials such as NSABP B-51/RTOG 1304 become available. Data from prospective cohort studies and retrospective reports show that treatment response affects the risk of locoregional recurrence $[10,11]$. However, although treatment response is a major prognostic factor, several other factors such as initial tumor and nodal stage, age, and tumor biology have to be accounted for [10]. Furthermore, the currently available data do not allow for conclusions regarding a predictive value of treatment response to adjuvant radiotherapy [12]. Whole-breast radiotherapy remains the standard of care after breast-conserving surgery. In patients with $\mathrm{cT} 1-2 \mathrm{cN}+$ and $\mathrm{pCR}$, post-mastectomy radiotherapy (and regional nodal irradiation in the case of breast-conserving surgery) should be considered and carefully discussed, especially in the case of further risk factors such as young age and aggressive tumor biology. Post-mastectomy radiotherapy is recommended in patients with locally advanced breast cancer regardless of treatment response.

Kühn: Most data regarding the effect of radiotherapy after neoadjuvant chemotherapy derive from retrospective and often single-center studies. Most of the data show a benefit for radiotherapy to the chest wall (in patients who undergo mastectomy) and the regional lymph nodes even in patients with a good response to chemotherapy. Available data are, however, very heterogenous and difficult to interpret. Mamounas et al. [11] analyzed the results from B18 and B27 and found negligible locoregional recurrence rates for patients with pCR (breast and lymph nodes). However, no data from prospective trials are available to support this hypothesis. The ongoing NSABP B-51 trial addresses this issue. Two European randomized trials (ATNEC and INDAX / EUBREAST 2) are in preparation.

Loibl: In the majority of cases, radiotherapy of the nodes.

\section{Participants}

Prof. Dr. med. Andreas Schneeweiss

Nationales Zentrum für Tumorerkrankungen

Sektion Gynäkologische Onkologie

Universitätsklinikum Heidelberg

Im Neuenheimer Feld 460

69120 Heidelberg, Germany

andreas.schneeweiss@med.uni-heidelberg.de

Prof. Dr. med. David Krug

Klinik für Strahlentherapie (Radioonkologie)

Universitätsklinikum Schleswig-Holstein

Campus Kiel

Arnold-Heller-Straße 3

24105 Kiel, Germany

david.krug@uksh.de

Prof. Dr. Thorsten Kühn

Frauenklinik

Städtische Kliniken Esslingen a.N.

Hirschlandstr. 97

73730 Esslingen, Germany

t.kuehn@klinikum-esslingen.de

Prof. Dr. med. Sibylle Loibl

Chair of the German Breast Group

GBG Forschungs $\mathrm{GmbH}$

Martin Behaim Strasse 12

63263 Neu-Isenburg, Germany

Sibylle.Loibl@GBG.de 


\section{References}

1 Gluz O, Nitz U, Liedtke C, Christgen M, Grischke EM, Forstbauer H, et al. Comparison of Neoadjuvant Nab-Paclitaxel+ Carboplatin vs Nab-Paclitaxel+Gemcitabine in Triple-Negative Breast Cancer: Randomized WSG-ADAPT-TN Trial Results. J Natl Cancer Inst. 2018 Jun;110(6):628-37.

2 Sharma P, López-Tarruella S, García-Saenz JA, Khan QJ, Gómez HL, Prat A, et al. Pathological Response and Survival in Triple-Negative Breast Cancer Following Neoadjuvant Carboplatin plus Docetaxel. Clin Cancer Res. 2018 Dec;24(23):5820-9.

3 Loibl S, O'Shaughnessy J, Untch M, Sikov WM, Rugo HS, McKee MD, et al. Addition of the PARP inhibitor veliparib plus carboplatin or carboplatin alone to standard neoadjuvant chemotherapy in triple-negative breast cancer (BrighTNess): a randomised, phase 3 trial. Lancet Oncol. 2018 Apr;19(4):497-509.

4 Sikov WM, Berry DA, Perou CM, Singh B, Cirrincione CT, Tolaney SM, et al. Impact of the addition of carboplatin and/or bevacizumab to neoadjuvant once-per-week paclitaxel followed by dose-dense doxorubicin and cyclophosphamide on pathologic complete response rates in stage II to III triple-negative breast cancer: CALGB 40603 (Alliance). J Clin Oncol. 2015 Jan;33(1):13-21.
5 Loibl S, Weber KE, Timms KM, Elkin EP, Hahnen E, Fasching PA, et al. Survival analysis of carboplatin added to an anthracycline/ taxane-based neoadjuvant chemotherapy and HRD score as predictor of response-final results from GeparSixto. Ann Oncol. 2018 Dec; 29(12):2341-7.

6 Sikov WM, Polley MY, Twohy EL, Perou CM, Singh B, Berry DA, et al. CALGB (Alliance) 40603: long-term outcomes (LTOs) after neoadjuvant chemotherapy (NACT) +/- carboplatin $(\mathrm{Cb})$ and bevacizumab (Bev) in triplenegative breast cancer (TNBC). J Clin Oncol. 2019 May;37 15_suppl:591.

7 Masuda N, Lee SJ, Ohtani S, Im YH, Lee ES, Yokota I, et al. Adjuvant Capecitabine for Breast Cancer after Preoperative Chemotherapy. N Engl J Med. 2017 Jun;376(22):214759.

8 Woodward WA, Fang P, Arriaga L, Gao H, Cohen EN, Reuben JM, et al. A phase 2 study of capecitabine and concomitant radiation in women with advanced breast cancer. Int $J$ Radiat Oncol Biol Phys. 2017 Nov;99(4):777-83.
9 Caudle AS, Yang WT, Krishnamurthy S, Mittendorf EA, Black DM, Gilcrease MZ, et al. Improved Axillary Evaluation Following Neoadjuvant Therapy for Patients With Node-Positive Breast Cancer Using Selective Evaluation of Clipped Nodes: Implementation of Targeted Axillary Dissection. J Clin Oncol. 2016 Apr;34(10):1072-8.

10 Krug D, Baumann R, Budach W, Dunst J, Feyer P, Fietkau R, et al.; Breast Cancer Expert Panel of the German Society of Radiation Oncology (DEGRO). Neoadjuvant chemotherapy for breast cancer-background for the indication of locoregional treatment. Strahlenther Onkol. 2018 Sep;194(9):797-805.

11 Mamounas EP, Anderson SJ, Dignam JJ, Bear HD, Julian TB, Geyer CE Jr, et al. Predictors of locoregional recurrence after neoadjuvant chemotherapy: results from combined analysis of National Surgical Adjuvant Breast and Bowel Project B-18 and B-27. J Clin Oncol. 2012 Nov;30(32):3960-6

12 Krug D, Baumann R, Budach W, Dunst J, Feyer P, Fietkau R, et al. Individualization of post-mastectomy radiotherapy and regional nodal irradiation based on treatment response after neoadjuvant chemotherapy for breast cancer : A systematic review. Strahlenther Onkol. 2018 Jul;194(7):607-18. 